International Workshop on Biopolymers: Thermodynamics, Kinetics and Mechanics of DNA, RNA and Proteins, 30.05-3.06.2005, The Abdus Salam International Centre for Theoretical Physics (ICTP), Trieste, Italy

\title{
On the perfect hexagonal packing of rods
}

\author{
E L Starostin \\ Department of Civil and Environmental Engineering, University College \\ London, Gower Street, London WC1E 6BT, UK \\ E-mail: ucesest@ucl.ac.uk
}

\begin{abstract}
In most cases the hexagonal packing of fibrous structures or rods extremizes the energy of interaction between strands. If the strands are not straight, then it is still possible to form a perfect hexatic bundle. Conditions under which the perfect hexagonal packing of curved tubular structures may exist are formulated. Particular attention is given to closed or cycled arrangements of the rods like in the DNA toroids and spools. The closure or return constraints of the bundle result in an allowable group of automorphisms of the cross-sectional hexagonal lattice. The structure of this group is explored. Examples of an open helical-like and closed toroidal-like bundles are presented. An expression for the elastic energy of a perfectly packed bundle of thin elastic rods is derived. The energy accounts for both the bending and torsional stiffnesses of the rods. It is shown that equilibria of the bundle correspond to solutions of a variational problem formulated for the curve representing the axis of the bundle. The functional involves a function of the squared curvature under the constraints on the total torsion and the length. The Euler-Lagrange equations are obtained in terms of curvature and torsion and due to the existence of the first integrals the problem is reduced to the quadrature. The $3 \mathrm{D}$ shape of the bundle may be readily reconstructed by integration of the Ilyukhin-type equations in special cylindrical coordinates. The results are of universal nature and are applicable to various fibrous structures, in particular, to intramolecular liquid crystals formed by DNA condensed in toroids or packed inside the viral capsids.
\end{abstract}

Submitted to: J. Phys.: Condens. Matter 


\section{Introduction}

It is known that the densest packing of infinite straight cylinders is hexagonal when all their axes are parallel [4]. It evidently corresponds to hexagonal packing of discs in a plane. The hexagonal packing of tubular objects (rods) occurs in numerous instances at scales ranging from nano to macro. Among examples there are nanotubes [47], high density columnar hexatic liquid crystalline DNA mesophases [33, 10, 32], actin bundles [48] and others. In most cases, this packing extremizes the interaction energy between filaments. Geometrically, it means that all pairs of neighbouring axes are located at constant distance to each other.

In some instances, the filaments are not straight. Then, a natural question arises of whether it is still possible to reach the same maximal density of packing. If yes, then the second question can be formulated as: what is the set of configurations of infinite (or closed) tubes that have the maximal density? By a tube (or a tubular neighbourhood) here we understand the set of all points in space whose distance from the smooth axial curve does not exceed the constant thickness radius. We can set the scale by fixing this radius to 1 . Moreover, we will assume that the global curvature of the axes is less or equal to 1 . Thus, the tubes cannot overlap, but they are perfectly flexible. It will be shown in the following that the densest packing class includes curvilinear axes, which should be relatively parallel. This implies that an arbitrary small twist of one axis around another immediately destroys the perfect hexagonal packing.

In most cases, one is interested in the optimal packing in some particular domain. In this paper we consider neither the geometrical properties of these boundaries nor the structural disturbances caused by their presence. The packing will be considered as optimal if in any section orthogonal to the axis of a tube at some point $P$, the crosssectional discs are hexagonally packed within a connected domain which contains the point $P$. In this sense, any single curvilinear perfect tube, which does not contact itself is optimally packed. This degenerate case just shows that the set of such locally optimal hexagonal packs is richer then the global packing.

Complicated spatial structures arise when the tubes are in contact with themselves. Important examples are intramolecular liquid crystals formed as a result of condensation of DNA in toroids [13, 36, 6, 17, 20] or a DNA arrangement inside viral capsids $[11,31,9]$. In this paper, particular attention is given to closed or cycled bundles of hexagonally packed rods. The closedness condition imposes a severe constraint on the whole structure. Indeed, take an orthogonal cross-section of the bundle. Then, what plays a role is the mapping of the $2 \mathrm{D}$ hexagonal lattice in the cross-section onto itself. The automorphisms that preserve both the distances and the connectivity form a discrete infinite group. Its study results in characterization of all possible closed hexagonally packed bundles: the writhing number [15] of each axis that realizes the mapping should equal $n / 6$, where $n$ is integer. One consequence of the automorphism group structure is that it is impossible to form a closed hexagonally packed bundle with a single filament: frustration is inevitable [38,6]. Examples of closed bundles made up with several strands are presented and the inverse spool model of the DNA packing inside a viral capsid is briefly discussed.

Clarification of the geometrical features is accompanied by consideration of mechanical properties. Assuming that the strands may be characterized as thin uniform isotropic elastic rods (a conventional model for DNA at mesoscale [49]), we compute the elastic energy of the bundle. A continuum limit case of circular 
cross-section of the bundle is studied in more detail. We show that the equilibria of the bundle may be described as extremal solutions to a variational problem for the axial curve in space. The functional is represented as a linear combination of three integrals: the first is a function of the squared curvature, the second is the total torsion and the last the length. This formulation makes it possible to apply the procedure for derivation of the Euler-Lagrange equations in terms of curvature and torsion [8]. The property that the functional involves only integral torsion and torsion is not coupled with curvature allows one to reduce the problem to the quadrature. With knowledge of curvature and torsion the spatial shape of the bundle may be readily computed by integration of the Ilyukhin-type equations in the specially chosen cylindrical coordinates $[29,21,22]$ which are widely used in the statics of rods.

\section{Geometry of unconstrained tube packing}

We start with consideration of a perfect tube of some length $\mathcal{L}$ with axis $\mathbf{r}(s), s$ being the arc length parameter. Let the tube be in a continuous contact with the maximal allowed number of other tubes of the same thickness. This number equals six [45], thus it may be said that the tubes are hexagonally packed. Denote the axes of the neighbouring tubes by $\mathbf{r}_{j}(s), j=1, \ldots, 6$. We can choose the same parametrization for all the tubes so that, for every $s$, the points $\mathbf{r}_{j}(s), j=1, \ldots, 6$, are the closest to the central axis $\mathbf{r}(s)$ and they lie in the vertices of a regular triangular lattice. Note that $s$ is not obliged to be an arc coordinate for $\mathbf{r}_{j}(s), j=1, \ldots, 6$. The vector field $\mathbf{m}_{j}(s) \equiv \mathbf{r}_{j}(s)-\mathbf{r}(s)$ is relatively parallel [5]. It implies that there is no twist of vectors $\mathbf{m}_{j}(s)$ about the central axis. We can add more layers of the tubes in the same manner as the first six tubes. Proceeding this way will allow us to build a bundle of parallel tubes that fill up some domain in space. The hexagonal packing provides the maximal density in this domain. In particular, if all the tubes are straight, we have the packing of cylinders [4].

Let us now obtain an equation that governs the position of the neighbouring tube for a given central axis. We omit the index $j$ for the sake of simplicity. Since $\|\mathbf{m}\|=\operatorname{const}(=2)$, we can write

$$
\frac{d \mathbf{m}}{d s}=\boldsymbol{\omega} \times \mathbf{m},
$$

and the vector $\boldsymbol{\omega}$ may be represented as $\boldsymbol{\omega}=\omega_{1} \mathbf{m}+\omega_{2} \mathbf{T} \times \mathbf{m}$ [45], where we denote by $\mathbf{T}=\frac{d \mathbf{r}}{d s}$ the tangent to the central axis.

By definition, the vector $\mathbf{m}$ connects the closest points on two curves, which implies $\mathbf{m} \cdot \mathbf{T}=0$. Differentiating this equation and further substitute (1) for $\frac{d \mathbf{m}}{d s}$, we come to $\omega_{2} \mathbf{m}^{2}=\mathbf{m} \cdot \frac{d \mathbf{T}}{d s}$, or, with the help of the Serret-Frenet equations, $\omega_{2} \mathbf{m}^{2}=\varkappa \mathbf{m} \cdot \mathbf{N}$, where $\mathbf{N}$ is the principal normal to $\mathbf{r}(s)$ and $\varkappa$ the curvature of this curve. Finally, the differential equation for the orientation vector $\mathbf{m}$ can be given the form

$$
\frac{d \mathbf{m}}{d s}=-\varkappa(\mathbf{m} \cdot \mathbf{N}) \mathbf{T}
$$

This is the main equation that describes the arrangement of tubes in the hexagonally packed bundle.

The infinite number of infinitely long cylinders can fill in the entire space. This is not possible for curvilinear tubes. Suppose the axis $\mathbf{r}(s)$ has the curvature $\varkappa_{0}>0$ and the torsion $\tau_{0}$ in some point $\mathbf{r}\left(s_{0}\right)$. The tangent $\mathbf{T}\left(s_{0}\right)$, the principal normal $\mathbf{N}\left(s_{0}\right)$ and 
the binormal $\mathbf{B}\left(s_{0}\right)$ form the orthonormal Frenet frame in the same point. The Frenet frames of all the tubes in given orthogonal cross-section are oriented identically. The normal plane $Q$ spanned by $\mathbf{N}\left(s_{0}\right)$ and $\mathbf{B}\left(s_{0}\right)$ is that of the orthogonal cross-section of the bundle. Now take some vector $\boldsymbol{\rho} \in Q$. Let $s_{1}$ be the arc coordinate for a tube's axis that passes through the point $\mathbf{r}_{1}=\mathbf{r}\left(s_{0}\right)+\boldsymbol{\rho}$. Then, $d s_{1}=h d s, h=1-\varkappa_{0} \boldsymbol{\rho} \cdot \mathbf{N}\left(s_{0}\right)$ and the curvature and the torsion of $\mathbf{r}_{1}$ are

$$
\varkappa_{1}=\varkappa_{0} / h \text { and } \tau_{1}=\tau_{0} / h
$$

respectively.

Since $\varkappa_{1} \leq 1$, we come to an inequality $\boldsymbol{\rho} \cdot \mathbf{N}\left(s_{0}\right) \leq \varkappa_{0}^{-1}-1$. In other words, all the axes of the tubes in the bundle may only cross the plane $Q$ in the region bounded by the straight line which is parallel to the binormal $\mathbf{B}\left(s_{0}\right)$ with the offset distance $\varkappa_{0}^{-1}-1$. Therefore, the thickness radius of the bundle with axis $\mathbf{r}(s)$ cannot exceed $\varkappa_{0}^{-1}$ in the direction of the principal normal. As the coordinate $s$ varies, the boundary straight line sweeps out a ruled surface which bounds a domain in space where the bundle can exist. Note that the maximal thickness of the bundle in the principal normal direction does not depend on the thickness of the individual tubes.

In one limiting case, we can consider a bundle made of a single tube of radius $R_{1}$, then the curvature of its axis cannot exceed $R_{1}^{-1}$. In other limit, the strands may be thought as being infinitesimally thin and an infinite number of them packed hexagonally inside a tubular domain of the same radius $R_{1}$. Again, the curvature of the axis of the tube is not allowed to be greater than $R_{1}^{-1}$. Note that, for this idealized model, once the bundle is bent at maximal rate (i.e. $\varkappa_{0}=R_{1}^{-1}$ ), the radius of curvature of a particular strand located at distance $R_{1}$ in the direction of $\mathbf{N}\left(s_{0}\right)$ goes to infinity. Nevertheless, as we will see in section 4, the elastic energy of this model remains finite.

Consider an integral torsion of two strands in the parallel bundle. Fix two orthogonal cross-sections at $s=s_{b}$ and $s=s_{e}$, then

$$
\int_{s_{b}}^{s_{e}} \tau_{0}(s) d s=\int_{s_{1 b}}^{s_{1 e}} \tau_{1}\left(s_{1}\right) d s_{1}, \quad s_{1 b}=s_{1}\left(s_{b}\right), s_{1 e}=s_{1}\left(s_{e}\right) .
$$

In other words, the total torsion is the same for an axis of every tube in the bundle.

For a closed curve $C$, the writhe (or the writhing number) is the double Gauß linking integral in the singular case of being taken over all pairs of points on the single curve $[14,15,24]$

$$
\mathcal{W} r(\mathbf{r})=\frac{1}{4 \pi} \int_{C} \int_{C} \frac{\left(\mathbf{r}\left(s_{1}\right)-\mathbf{r}\left(s_{2}\right)\right) \cdot\left(\mathbf{T}\left(s_{1}\right) \times \mathbf{T}\left(s_{2}\right)\right)}{\left\|\mathbf{r}\left(s_{1}\right)-\mathbf{r}\left(s_{2}\right)\right\|^{3}} d s_{1} d s_{2} .
$$

The writhe depends only on the shape of the curve. The writhe relates to the area swept out by the tangent indicatrix on the surface of unit sphere [16]. This makes it possible to define the writhe for non-closed curves [34, 43, 44]. Since the tangent indicatrix is identical for every strand in the bundle, they all have the same writhe.

By way of example let us take a look at the regular helical curve: $\mathbf{r}(s)=$ $\left(\cos a s, \sin a s, \sqrt{1-a^{2}} s\right), 0 \leq a \leq 1$. Equation (2) may be transformed to the system

$$
\frac{d \xi}{d s}=a \eta, \quad \frac{d \eta}{d s}=a\left(a^{2}-1\right) \xi, \quad \frac{d m_{z}}{d s}=a^{2} \sqrt{1-a^{2}} \xi,
$$


and the first two components of the vector $\mathbf{m}=\left(m_{x}, m_{y}, m_{z}\right)$ are expressed as $m_{x}=\xi \cos a s-\eta \sin a s, m_{y}=\xi \sin a s+\eta \cos a s$. The explicit solution of (5) is easy to find:

$$
\begin{aligned}
& \xi=c_{1} \cos \tau s+c_{2} \sin \tau s, \quad \eta=\sqrt{1-a^{2}}\left(c_{2} \cos \tau s-c_{1} \sin \tau s\right), \\
& m_{z}=a\left(c_{1} \sin \tau s-c_{2} \cos \tau s\right),
\end{aligned}
$$

where $\tau=a \sqrt{1-a^{2}}$ is the torsion and $c_{1}, c_{2}$ are the constants of integration such that $\mathbf{m}^{2}=c_{1}^{2}+c_{2}^{2}$. Figure 1 shows a bundle of six tubes arranged at constant distance from the central tube and from the neighbours. At every section which is orthogonal to all their axes, the crossing points form the hexagonal lattice. There is no cholesteric angle. This arrangement differs from the case when the strands are helices on the concentric cylinders, there are no cylindrical layers.

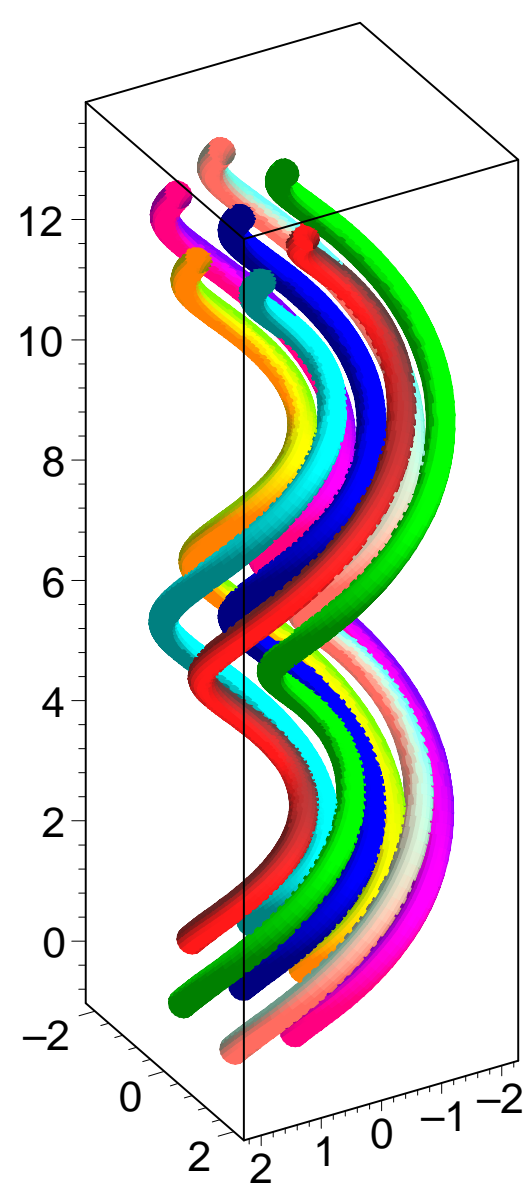

Figure 1. The perfectly packed bundle of $1+6$ tubes. The centreline of the core tube (shown in blue) is a regular helix. Each of the other six strands that surround the core, is a trace of the end of a vector $\mathbf{m}$, which is orthogonal to the core and parallel transported along the core according to (5). The tubes are shown thinner to ease representation.

It seems to be instructive to compare this example with the helical spool packing 
which has been long considered as one of the most likely models of arrangement of a double-stranded DNA inside a viral capsid [11, 12, 40, 18, 51, 9, 39]. Numerical modelling also seems to suggest that spool-like conformations may represent the stable states or they can naturally emerge in the packaging process $[25,1,35]$. Here we would like only to make clear some geometrical features inherent to layered spool-like structures as considered from the viewpoint of the parallel packing.

Imagine a helical tube in which the successive coils touch each other continuously. Fix a point on the axis of the tube and take an orthogonal cross-section of the tube at this point. It is easy to show that the plane of this section crosses all other coils non-orthogonally. But the hexagonal packing is only possible with parallel tangents to the axes at the cross-section [45]. Therefore, the packing in a cylindrical spool when the helical tubes form layers [37] is nowhere hexagonal. In other words, the adjacent coils cannot be made relatively parallel (cf. figure 1).

Furthermore, the spool formation scenario from outer to inner layers [37] poses more problems: 1) it is not clear how DNA may switch to a next layer without either self-intersection or sharp bends, depending on its relative direction in the adjacent layers; 2) since the radius of the helix decreases with the number of coils being the same in all the layers, the DNA tube is forced to be compressed in the inner layers, which should lead to frustration. On pictures of final conformations presented in Ref. [1] one can see that even the most outer layers are far from parallel packing, even the handedness of the helices differs. This causes the overhangs which in turn prompt the complete destruction of the layering in the inner domain. On the other hand, switching between layers by means of a short connection located in the core domain may provide better approximation to parallel packing, i.e. more compact structures, but such an arrangement seems difficult to reconcile with the packaging pathway.

\section{Cyclization of bundles}

We come to an analysis of the parallel bundles in which the strands touch themselves. Consider a bundle of tubes that are hexagonally packed. Take a plane $Q$ of an orthogonal cross-section of some tube with an axis point $P$. Let $\Psi \in Q$ be a connected domain which contains all the cross-sectional discs of tubes in the bundle touching each other. Clearly, this plane is orthogonal to all the axes of the tubes that cross $\Psi$. Thus, the points of the crossings are the vertices of a triangular lattice $i \mathbf{e}_{1}+j \mathbf{e}_{2}, i, j \in \mathbb{Z}$, and $\mathbf{e}_{1}^{2}=\mathbf{e}_{2}^{2}=2 \mathbf{e}_{1} \cdot \mathbf{e}_{2}=1$. Actually, (2) defines a parallel vector field in the $3 \mathrm{D}$ space and if a 2D lattice is specified in a plane orthogonal to the field, then the structure of this lattice remains invariant when the plane moves along the field. This observation opens the way to generalizations for tubes of different thickness as well as for arbitrary lattices.

Now we are interested in arrangements of tubes in 3D space such that, having started at some particular vertex, the axis comes back to the same plane either at the same point or at another vertex. Moreover, we will assume that the tubes originating from two neighbouring vertices will remain in continuous contact and thus return to neighbouring vertices. Then, the general question to be asked is: what is a set of allowable automorphisms of the lattice induced by the three-dimensional shape of the bundle?

Let us fix the origin of the reference frame in some vertex in the plane $Q$. Without loss of generality, we can focus on the tube with axis $\mathbf{r}(s), s \in[0, \mathcal{L}]$ that starts in the origin, we call it the core. Let it come back next time for $s=\mathcal{L}$ at the vertex 
$\Delta \mathbf{r}=k \mathbf{e}_{1}+l \mathbf{e}_{2}$, for some $k, l \in \mathbb{Z}$. Now take a neighbouring starting vertex $\mathbf{p}_{1}$; without loss of generality we can take $\mathbf{p}_{1}=\mathbf{e}_{1}$. It is enough to consider only one neighbour, because, if we know the shape of two neighbouring tubes, we can reconstruct the entire bundle uniquely, including the automorphism of the lattice. Let the neighbouring tube end up in the vertex $\mathbf{p}_{2}=\Delta \mathbf{r}+\Omega^{n} \mathbf{p}_{1}$, where $\Omega^{n}$ is a rotation linear operator with matrix

$$
\Omega^{n}=\left(\begin{array}{cc}
\cos \frac{\pi}{3} n & -\sin \frac{\pi}{3} n \\
\sin \frac{\pi}{3} n & \cos \frac{\pi}{3} n
\end{array}\right), \quad n \in \mathbb{Z}_{6},
$$

and $\Omega^{1} \mathbf{e}_{1}=\mathbf{e}_{2}, \Omega^{2} \mathbf{e}_{1}=\Omega^{1} \mathbf{e}_{2}=\mathbf{e}_{2}-\mathbf{e}_{1}, \Omega^{3} \mathbf{e}_{1}=-\mathbf{e}_{1}, \Omega^{4} \mathbf{e}_{1}=-\mathbf{e}_{2}, \Omega^{5} \mathbf{e}_{1}=\mathbf{e}_{1}-\mathbf{e}_{2}$, $\Omega^{0} \mathbf{e}_{1}=\mathbf{e}_{1}$.

Let us first consider the case when the core comes back to the origin, i.e., $k=l=0$. Then the mapping of the lattice onto itself is just a rotation through the angle $\frac{\pi}{3} n$ around the origin. If $n=0$, then the map is identity, all the tubes come back to their starting places. Thus the whole bundle contains a set of closed tubes and every tube crosses the domain $\Psi$ only once. Such a bundle is often used as a simplified model in studies of DNA condensation into nanostructures, in particular, toroids $[50,20]$ or spools $[40,39]$. For $n=1,5$, every tube makes closure after 6 intersections with $\Psi$ (figure 2(a)), for $n=2,4$ after three crossings (figure 2(b)) and for $n=3$ after two (figure 2(c)).

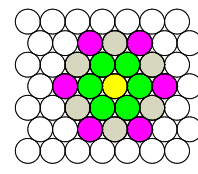

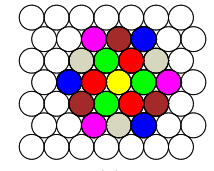

(b)

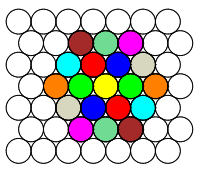

(c)

Figure 2. Cross-sectional lattice of the bundle. Each tube is marked with different colour so that the discs of the same colour represent a sequence of crossings for a particular tube. (a) Rotation of the lattice around the central disc through $\pm \frac{1}{3} \pi \bmod 2 \pi(n=1,5)$. (b) Rotation of the lattice around the central disc through $\pm \frac{2}{3} \pi \bmod 2 \pi(n=2,4)$. (c) Rotation of the lattice around the central disc through $\pm \pi \bmod 2 \pi(n=3)$.

Take the axes of the core and of a neighbouring tube and consider a closed thin ribbon which is formed by an arbitrary short vector $\epsilon \Delta \mathbf{r}(s), s \in[0, \mathcal{L}]$, pointing from the core axis to the closest point onto the neighbouring axis. The ribbon need not be closed; only one of its edges (the core axis) is closed. We denote by $\gamma$ the angle between the vectors $\Delta \mathbf{r}(0)$ and $\Delta \mathbf{r}(\mathcal{L})$ (figure 3). The twisting number of the ribbon (i.e., of the pair $(\mathbf{r}, \Delta \mathbf{r}))$ is defined by

$$
\mathcal{T} w(\mathbf{r}, \Delta \mathbf{r})=\frac{1}{2 \pi} \int_{0}^{\mathcal{L}} \frac{d \mathbf{r}}{d s} \times \Delta \mathbf{r} \cdot d(\Delta \mathbf{r}) .
$$

An equation holds

$$
\mathcal{T} w(\mathbf{r}, \Delta \mathbf{r})+\mathcal{W} r(\mathbf{r})+\frac{\gamma}{2 \pi}=0 \quad \bmod 1
$$

(see Appendix A in Ref. [43]).

The ribbon is untwisted, because of the parallelism of its edges. Then, the fractional part of the writhe of the closed core axis equals $-\frac{\gamma}{2 \pi}=-\frac{1}{2 \pi} \cdot \frac{\pi}{3} n=-\frac{n}{6}$ 


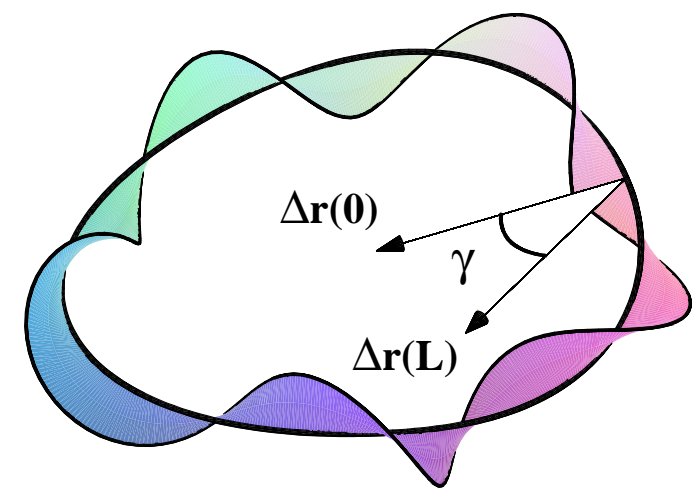

Figure 3. A non-closed ribbon based on smooth closed curve $\mathbf{r}$.

and, as already explained in section 2, the writhe of a piece of every axis between two intersections with $\Psi$ is the same. Note that, for $n \neq 0$, these pieces are not closed though they have parallel tangents at their ends.

Now we come to the more general case when $|k|+|l| \neq 0$. Our aim is to find a point $\mathbf{c}=\mu \mathbf{e}_{1}+\nu \mathbf{e}_{2}$ such that the rotation of the plane $Q$ through the angle $\frac{\pi}{3} n$ maps the origin into the point $\Delta \mathbf{r}$ and $\mathbf{p}_{1}$ into $\mathbf{p}_{2}$. We shall call this point $\mathbf{c}$ the centre of rotation and it should satisfy the equation

$$
\Omega^{n} \mathbf{c}=\mathbf{c}-\Delta \mathbf{r},
$$

which transforms into a system of two equations for the coordinates $\mu, \nu$. Equation (6) implies that $\Omega^{n}\left(\mathbf{c}-\mathbf{p}_{1}\right)=\mathbf{c}-\mathbf{p}_{2}$ which shows that the point $\mathbf{p}_{1}$ goes to $\mathbf{p}_{2}$ after rotation. This means that the transformation of the plane $Q$ is consistent with an automorphism of the lattice.

Consider now all the possible cases of various $n \in \mathbb{Z}_{6}$ and find the coordinates of the centre of rotation $\mathbf{c}$ explicitly. Note that the point $\mathbf{c}$ is not obliged to belong to the domain $\Psi$.

$\underline{n=0}$. The centre of rotation does not exist; the transformation is a translation along $\Delta \mathbf{r}$ (figure $4($ a)).

$\underline{n=1}$. The centre of rotation is located in a vertex of the triangular lattice:

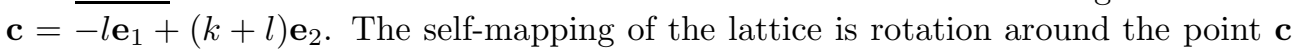
through $\frac{\pi}{3} \bmod 2 \pi$ (figure $2(\mathrm{a})$ ).

$\underline{n=2}$. The centre of rotation has the coordinates: $\mu=\frac{k-l}{3}, \nu=\frac{k+2 l}{3}$. It can be represented as $\mathbf{c}=\frac{\sigma}{3}\left(\mathbf{e}_{1}+\mathbf{e}_{2}\right)+s \mathbf{e}_{1}+t \mathbf{e}_{2}$ with $\sigma=0, \pm 1$ and $s, t \in \mathbb{Z}$, from where we see that the centre of rotation is located in a vertex of a reciprocal lattice. If $l-k=3 h, h \in \mathbb{Z}(\sigma=0)$, then the centre of rotation coincides with the centre of a cross-sectional disc of a tube which closes after one cycle (figure 2(b)). Otherwise, the point $\mathbf{c}$ is located in the centre of one of the equilateral triangles of the initial lattice. There exists no core tube, instead, all the lattice is decomposed into triples of discs and every triple corresponds to a single tube which closes after three intersections with $\Psi$ (figure 4(b)). 


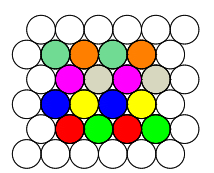

(a)

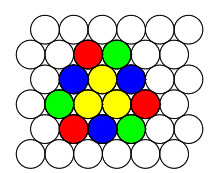

(b)

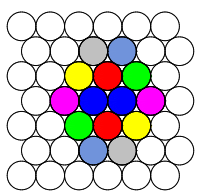

(c)

Figure 4. Cross-sectional lattice of the bundle. Each tube is marked with different colour so that the discs of the same colour represent a sequence of crossings for a particular tube. (a) Translation of the lattice along $\mathbf{e}_{1}(n=0)$.

(b) Rotation of the lattice around $\frac{1}{3}\left(\mathbf{e}_{1}+\mathbf{e}_{2}\right)$ through $\pm \frac{2}{3} \pi \bmod 2 \pi(n=2,4)$.

(c) Rotation of the lattice around $\frac{1}{2} \mathbf{e}_{1}$ through $\pm \pi \bmod 2 \pi(n=3)$.

$\underline{n=3}$. The lattice map is a rotation through $\pi \bmod 2 \pi$ around $\mathbf{c}=\frac{1}{2}\left(k \mathbf{e}_{1}+l \mathbf{e}_{2}\right)$. If $k$ and $l$ are both even, then the map is as in figure 2(c). Otherwise, there is no self-joining core, all the tubes have two crossings with $\Psi$ (figure 4(c)).

$\underline{n=4}$. This case resembles that for $n=2$. The lattice is rotated through $\frac{4}{3} \pi$ $\bmod 2 \pi$ around $\mathbf{c}=\frac{1}{3}\left((2 k+l) \mathbf{e}_{1}+(l-k) \mathbf{e}_{2}\right)=\frac{\sigma}{3}\left(\mathbf{e}_{1}+\mathbf{e}_{2}\right)+s \mathbf{e}_{1}+t \mathbf{e}_{2}$ with $\sigma=0, \pm 1$, $s, t \in \mathbb{Z}$. If $l-k=3 h, h \in \mathbb{Z}(\sigma=0)$, then the point $\mathbf{c}$ lies in the centre of the disc of the one-cycle core (figure 2(b)). Otherwise, there exist only cycles of period three (figure 4(b)).

$\underline{n=5}$. This last case is similar to that for $n=1$. The lattice is mapped onto itself by rotation through $\frac{5}{3} \pi \bmod 2 \pi$ around the centre $\mathbf{c}=(k+l) \mathbf{e}_{1}-k \mathbf{e}_{2}$.

Summing up, we can say that the automorphism group of the lattice is finitely generated by the following set of transformations:

1) an identity map,

2) translations along the lattice vectors $\mathbf{e}_{1}$ and $\mathbf{e}_{2}$,

3) a rotation through $\frac{1}{3} \pi$ around the origin,

4) a rotation through $\frac{2}{3} \pi$ around $\frac{1}{3}\left(\mathbf{e}_{1}+\mathbf{e}_{2}\right)$,

5) a rotation through $\pi$ around $\frac{1}{2} \mathbf{e}_{1}$.

The above group includes all the transformations of the lattice which may be realized with a bundle of continuously hexagonally packed tubes. We see that the maximal number of returns of a single closed tube is six. This implies that a thick (multi-layered) closed bundle may be formed only by a set of separate closed tubes. Note that in the case of translations, one may speak only about self-touching bundles, not closed in a strict sense.

The spatial configuration of the bundle determines the continuous mapping of the domain $\Psi$ of the cross-sectional plane which moves in space as the parameter $s$ varies. Therefore, the centres of rotation (when exist) form a closed curve $\mathbf{c}(s)$, which we call the axis of the bundle. As in the above-considered particular case, the fractional part of the writhe of a piece of axis between consecutive crossings equals $-\frac{n}{6}$. To prove this in case $n \neq 0$, one has to analyze a ribbon formed by a vector $\epsilon \Delta \mathbf{r}(s)$ directed from the axis $\mathbf{c}(s)$ to the closest point onto the neighbouring axis.

If $n=0$, then we can consider a ribbon formed by the vector $\epsilon \Delta \mathbf{r}(s)$ moving along an open or closed axis of some tube on the interval between two consecutive intersections with $\Psi$. The ribbon is untwisted and both its edges may not be closed, but the orientation of its ends is the same. If open, such a ribbon may be made closed by adding another untwisted ribbon, hence its writhe (i.e., the writhe of the tube's 
axis) should be integer (details on how to deal with the writhe of open curves can be found in Refs. [43, 44]).

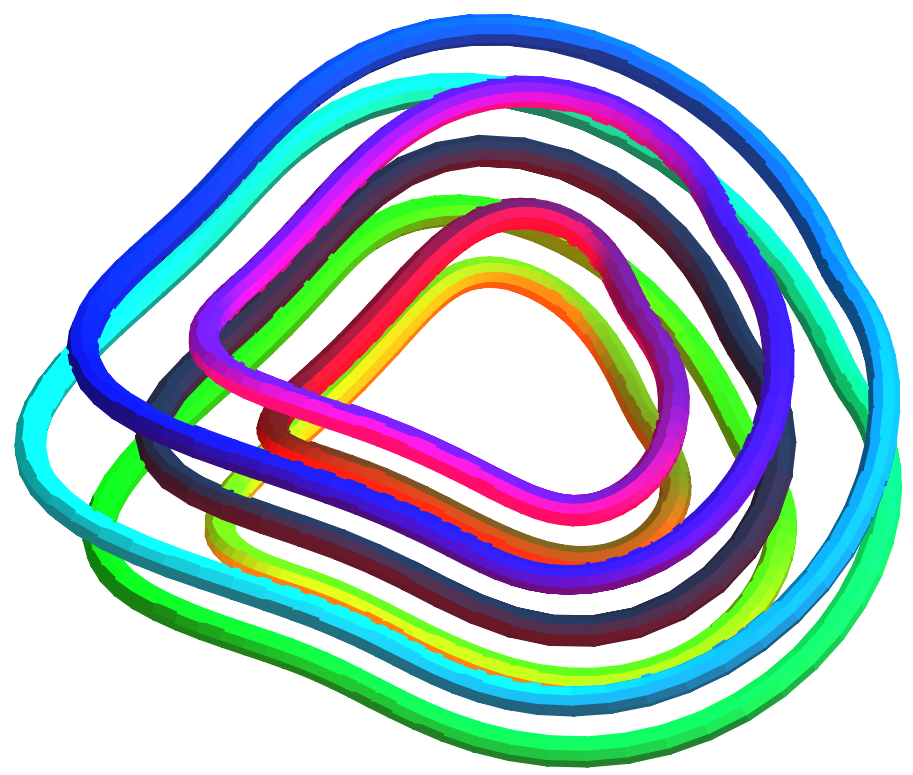

Figure 5. The perfectly packed bundle made up of two closed tubes. The core (dark) makes one turn and the second tube winds six times. The colour variation codes the arc length. The tubes are shown thinner to ease representation.

An example of a perfectly packed closed bundle is shown in figure 5 . The axis of the central tube lies on the surface of a torus. It closes after one turn around the torus hole. The writhing number of the central axis was made equal to $-\frac{1}{6}$. The second tube winds six times around the torus hole, forming the hexagonally packed structure. Another example is presented in figure 6 , it corresponds to the lattice transformation of figure 4(b). The mapping of figure 4(c) takes place in the bundle shown in figure 7 . Three closed tubes are drawn. The axis of each tube may be considered as an edge of a Möbius strip. The contact line of the central tube lies on the surface of a torus and its writhing number is $-\frac{1}{2}$.

Consider a closed perfect bundle made with a number of closed components. All the components of the bundle intersect every orthogonal cross-section the same number of times; we call this number $p$ the winding number. (The only exception may be the core component which may coincide with the axis $\mathbf{c}(s)$ : it always has one point of intersection by definition.) If $\{|\mathcal{W} r(\mathbf{c})|\}=n / 6, n \in \mathbb{Z}_{6}$, then $p(n)$ is defined as follows: $p(0)=1$ and $p(n)=6 / \min \{n, 6-n\}, n \neq 0$.

Let us first examine the case when the mapping of a cross-section is a rotation and the axis of the bundle $\mathbf{c}(s)$ ( $s$ is the arc length) is defined. We denote the intersections points $\mathbf{m}_{i}(s), i=1, \ldots, p$, for a non-core component. The cross-section of each component is $p$-fold central symmetric with respect to $\mathbf{c}(s)$ for every $s$. Hence, $\mathbf{m}_{i}(s)=\Omega^{\frac{6 i}{p}} \mathbf{m}_{1}(s)$ and

$$
\sum_{i=1}^{p} \mathbf{m}_{i}(s)=\mathbf{0}
$$




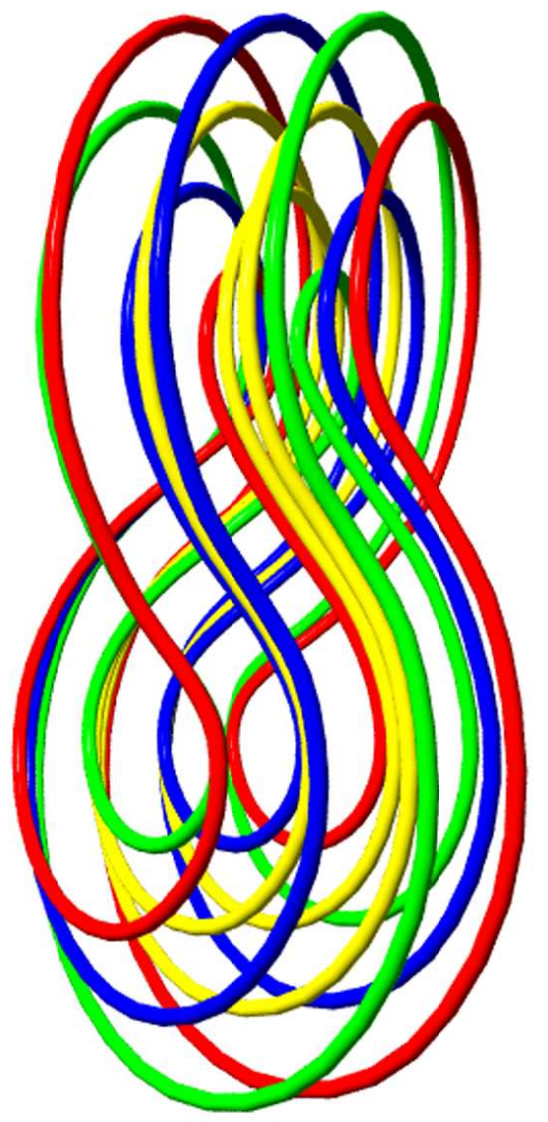

Figure 6. The perfectly packed bundle made up of four closed tubes. The tubes are shown thinner to ease representation.
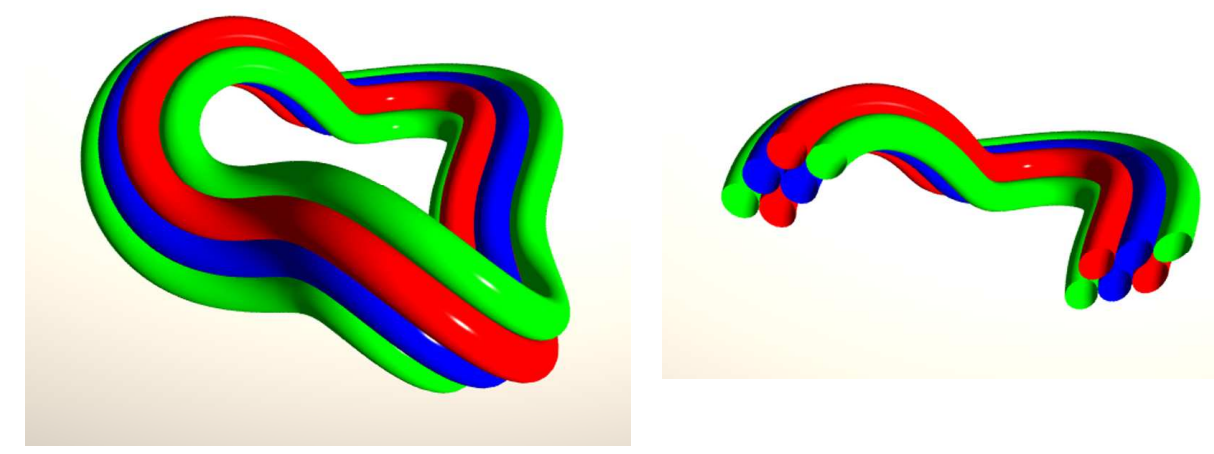

Figure 7. The perfectly packed bundle that corresponds to the case of figure 4(c). 
Let $\mathcal{L}$ be the length of the axis $\mathbf{c}(s)$, i.e., the length of the core component, if the latter exists,

$$
\mathcal{L}=\oint d s
$$

Compute the length of one component between its consecutive crossings of a given cross-section $Q$

$$
l_{m i}=\int d s_{m i}=\int\left(1-\varkappa(s)\left(\mathbf{m}_{i}(s) \cdot \mathbf{N}(s)\right)\right) d s,
$$

where $\varkappa(s)$ is the curvature of the axis $\mathbf{c}(s)$ and $\mathbf{N}(s)$ its principal normal. Now we can find the total length of the $p$-th component

$$
\mathcal{L}_{p}=\sum_{i=1}^{p} l_{m i}=\oint\left(p-\varkappa(s)\left(\left(\sum_{i=1}^{p} \mathbf{m}_{i}(s)\right) \cdot \mathbf{N}(s)\right)\right) d s=p \oint d s=p \mathcal{L} .
$$

Thus, we have shown that the total length of every component is conserved and is a multiple of the length of the axis of the bundle; the integer coefficient is determined by the topology of the bundle. This property of the closed parallel bundle makes it possible to vary the shape of the bundle in space while retaining its hexagonal packing, though sliding of the neighbouring tubes is allowed.

We may suggest that the perfect bundle can be built from pieces of DNA (or other polymer) molecules with their lengths quantized which can be done by dimerization, though it could be difficult to properly link the closed dimers to each other before condensation. Still, it seems that the necessary technique already exists and may be used to obtain the curvilinear closed liquid crystalline bundles of geometrically maximal density of long and stiff polymers, particularly, of DNA [6, 41, 19]. If one managed to create a set of properly interlinked closed strands, then the geometry of their most compact state would be the parallel packing in a bundle with predefined writhe, which results in a constraint on the shape of the bundle in space. In other words, the topological structure of the bundle manifests itself in the 3D shape.

We have excluded the case of identity map from the above computation of the length. Now consider this arrangement of closed rods where each rod closes to itself after single cycle. (Note that this condition does not imply that the rods are not linked.) Take some tube and define it as the bundle's axis of length $\mathcal{L}$. Let another rod have the intersections points $\mathbf{m}_{q}(s)$. Note that there is always room for a rod that has intersections at $-\mathbf{m}_{q}(s)$. It is easy to see now that the sum of their lengths equals $2 \mathcal{L}$. Thus, if the cross-section of the bundle is centrally symmetric, then all the components except the central one (if it exists) may be arranged in pairs and the total length of every pair is $2 \mathcal{L}$.

\section{Conformational energy}

In this section we consider an idealized model of a perfect bundle in the continuum limit with the director field $\mathbf{T}$. Then we can write out the elastic Frank-Oseen energy density of liquid crystals for mesomorphic phases which has the form [24, 26]

$$
\begin{aligned}
& f_{F O}=\frac{1}{2} K_{1}[\nabla \cdot \mathbf{T}]^{2}+\frac{1}{2} K_{2}[\mathbf{T} \cdot(\nabla \times \mathbf{T})]^{2}+\frac{1}{2} K_{3}[\mathbf{T} \times(\nabla \times \mathbf{T})]^{2}+ \\
& +K_{13} \nabla \cdot[(\nabla \cdot \mathbf{T}) \mathbf{T}]-K_{24} \nabla \cdot[(\nabla \cdot \mathbf{T}) \mathbf{T}+\mathbf{T} \times(\nabla \times \mathbf{T})],
\end{aligned}
$$


where $K_{i}, i=1,2,3$, and $K_{13}, K_{24}$ are elastic constants.

For perfect hexagonal packing, $\nabla \cdot \mathbf{T}=0$ and there are neither first splay term in (8) nor the splay-bend term with coefficient $K_{13}$. It is easy to see that the second twist term also vanishes. The last saddle-splay term is proportional to the Gaussian curvature of the surface to which the director $\mathbf{T}$ is normal [26]. In our case, this surface is a plane of cross-section with no curvature. Therefore, the energy density (8) reduces to the bending term $\frac{1}{2} b \varkappa^{2}$, with $b \equiv K_{3}$ being the bending rigidity density. (Note that the above reduction of energy is likely to be considered as a proper approximation for materials when $K_{3}$ significantly exceeds the other elastic moduli which is often the case [26].)

In the further analysis, we assume that the cross-section $\Psi$ of the bundle is a circle of radius $R$. Let the axis of the bundle be $\mathbf{c}(s), s$ the arc length, $s \in[0, \mathcal{L}]$. We also assume that the Frenet frame is well defined with the principal normal $\mathbf{N}$ and the binormal B. The elastic energy of each strand is computed as the bending and torsion energy of a thin isotropic uniform elastic rod of circular cross-section of radius $R$. All the rods in the bundle have identical mechanical properties and the same thickness. Our model does not account for a specific interaction energy between rods in the bundle.

\subsection{Energy of bending}

The bending energy of the bundle is proportional to the integral of the squared curvature $\tilde{\varkappa}$ of the director field, taken over the volume of the bundle $V$

$$
E_{\text {bend }}=\frac{b}{2} \iiint_{V} \tilde{\varkappa}^{2} d v=\frac{b}{2} \iint_{\Psi} \int_{0}^{l_{m}} \varkappa_{m}^{2}\left(s_{m}\right) d s_{m} d \sigma
$$

where $d \sigma$ is the area element of the cross-section and $s_{m}$ is the arc coordinate of the strand specified in the cross-sectional plane by vector $\boldsymbol{\rho}=\rho(\mathbf{N} \cos \varphi+\mathbf{B} \sin \varphi)$, $\|\boldsymbol{\rho}\|=\rho \leq R, \varphi \in[0,2 \pi]$. Thus, $\rho$ and $\varphi$ are polar coordinates in the cross-section. If $\varkappa(s)$ is the curvature of the axis $\mathbf{c}(s)$, then by using (3), the bending energy (9) may be given the form

$$
E_{\text {bend }}=\frac{b}{2} \iint_{\Psi} \int_{0}^{\mathcal{L}} \frac{\varkappa^{2}(s)}{1-\varkappa(s) \boldsymbol{\rho} \cdot \mathbf{N}} d s d \sigma,
$$

or, in polar coordinates,

$$
E_{\text {bend }}=\frac{b}{2} \int_{0}^{\mathcal{L}} \varkappa^{2}(s) \int_{0}^{2 \pi} \int_{0}^{R} \frac{\rho}{1-\varkappa(s) \rho \cos \varphi} d \rho d \varphi d s .
$$

Integration of the last formula leads to

$$
E_{\text {bend }}=\pi b \int_{0}^{\mathcal{L}}\left(1-\sqrt{1-R^{2} \varkappa^{2}(s)}\right) d s .
$$

In particular, if the bundle is a torus of major radius $P$ (all the strands that form the bundle simply have circular axes), then $\varkappa(s)=\frac{1}{P}, \mathcal{L}=2 \pi P$ and $E_{\text {bend }}=2 \pi^{2} b\left(P-\sqrt{P^{2}-R^{2}}\right)$. 
If $R \varkappa(s) \ll 1$, then the bundle may be considered as relatively thin (compared to the curvature of the centreline). Then (10) clearly allows for an approximation

$$
E_{\text {bend }} \approx \pi R^{2} \frac{b}{2} \int_{0}^{\mathcal{L}} \varkappa^{2}(s) d s,
$$

which is the bending energy of the thin rod with the bending stiffness $B=\pi R^{2} b$.

Thus, the bending stiffness of the bundle is proportional to the number of strands it consists of, not to the square of this number as it would be if the bundle were a thick rod made of homogeneous material [29]. The presence of cross-links between filaments (e.g., in the actin fiber) causes the similar effect [42]. The elastic properties of some other bundles like neurofilaments do not show the influence of cross-linking ([7], p. 295).

\subsection{Energy of torsion}

For a thin elastic rod of circular cross-section and which is not under action of a distributed moment with a non-zero tangential component, there exists an invariant $C(s)\left(\omega_{1}(s)-\omega_{10}(s)\right)=$ const, where $C(s)$ is the torsional rigidity, $\omega_{1}(s)$ is the tangential component of the Darboux vector and $\omega_{10}(s)$ is this component in the relaxed state. We have already assumed that, for each strand in the bundle, $\omega_{10}(s)=$ const, $C(s)=C=$ const. This implies $\omega_{1}=$ const.

To find the torsional energy of the bundle, we have to integrate over all the rods

$$
\begin{aligned}
& E_{\text {tors }}=\frac{c}{2} \iiint_{V}\left(\tilde{\omega}_{1}-\tilde{\omega}_{10}\right)^{2} d v=\frac{c}{2} \iint_{\Psi} \int_{0}^{l_{m}}\left(\omega_{1 m}-\omega_{10 m}\right)^{2} d s_{m} d \sigma= \\
& =\frac{c}{2} \iint_{\Psi} \frac{1}{p} \int_{0}^{\mathcal{L}_{p}}\left(\omega_{1 m}-\omega_{10 m}\right)^{2} d s_{m} d \sigma=\frac{c}{2} \mathcal{L} \iint_{\Psi}\left(\omega_{1 m}-\omega_{10 m}\right)^{2} d \sigma,(11)
\end{aligned}
$$

where $c=\frac{C}{\pi R^{2}}$ is the torsional rigidity density. Note that the twist rates may differ for different components. For simplicity, we will assume that the rods are not twisted in their relaxed state $\left(\omega_{10 m}=0\right)$.

\section{Equilibria of closed perfect bundle}

Consider a closed bundle made up with symmetric rods, each rod being also closed, for a given self-mapping of a cross-section. Thus, the topology of the bundle is fixed. We specify the boundary value problem by imposing the periodical conditions for every rod in the bundle. These conditions include both the closure of the centrelines of each component and of their material frames. A stable equilibrium shape of the bundle corresponds to a minimum of the total elastic energy $E=E_{\text {bend }}+E_{\text {tors }}$ among all parallel bundles with axes of a given length satisfying the boundary conditions at the ends.

The variations of the shape preserve the total torsion of every rod. If the axis of a bundle is an extremal for $E$, then it is an extremal for the $E_{b e n d}$ for fixed $\omega_{1 m}$. Furthermore, it is an extremal for a linear combination $\lambda_{1} \mathcal{K}+\lambda_{2} \mathcal{T}$ (cf. [30]), where 
$\mathcal{T}=\oint \tau d s$ is the total torsion and $\mathcal{K}=\oint f\left(\varkappa^{2}\right) d s$ with $f\left(\varkappa^{2}\right)$ defined as the integrand in Eq. (10).

As shown in Section 3, the shape variation of the perfect bundle with centreline of fixed length does not affect the lengths of the components (or pairs of them in case of a non-rotational map). Hence, we can impose the length constraint and take into consideration the centrelines with arbitrary regular parametrization. Thus, we come to the generalization of Theorem 1 from Ref. [30] to the parallel bundles:

The centreline of a parallel bundle of circular cross-section made up with isotropic uniform thin elastic rods in equilibrium is an extremal for a linear combination of three integrals

$$
\mathcal{F}=\lambda_{1} \mathcal{K}+\lambda_{2} \mathcal{T}+\lambda_{3} \mathcal{L}
$$

Clearly, setting $f\left(\varkappa^{2}\right)=\varkappa^{2}$ and keeping only one component reduce the above statement to the mentioned Theorem 1 for a single rod. That theorem allows for the converse [23]. Now we show that the converse exists for the parallel bundle, too.

Let $\mathbf{r}(s)$ be a closed extremal of (12) of length $\mathcal{L}$ with writhe $\mathcal{W} r=n / 6, n \in \mathbb{Z}$. Thus, the curve $\mathbf{r}(s)$ generates a parallel vector field with winding number $p$. All the vector lines are closed and, based on any of them, say, on $\mathbf{r}_{m}(s)$, we can construct a closed thin rod with constant twist rate $\omega_{1 m}$ such that

$$
\omega_{1 m} \mathcal{L}_{p}=\oint_{0}^{\mathcal{L}_{p}} \tau_{m} d s_{m}+2 \pi j_{m}, \quad j_{m} \in \mathbb{Z} .
$$

Consider the elastic energy of the rod

$$
E_{m}=\oint_{0}^{\mathcal{L}_{p}}\left(\frac{b}{2} \varkappa_{m}^{2}\left(s_{m}\right)+\frac{c}{2} \omega_{1 m}^{2}\right) d s_{m}
$$

and its variation on the set of closed rods of the same length

$$
\delta E_{m}=\frac{b}{2} \delta \oint_{0}^{\mathcal{L}_{p}} \varkappa_{m}^{2}\left(s_{m}\right) d s_{m}+\frac{c}{2} \delta \oint_{0}^{\mathcal{L}_{p}} \omega_{1 m}^{2} d s_{m}=\frac{b}{2} \delta \oint_{0}^{\mathcal{L}_{p}} \varkappa_{m}^{2}\left(s_{m}\right) d s_{m}+c \omega_{1 m} \delta \oint_{0}^{\mathcal{L}_{p}} \omega_{1 m} d s_{m} .
$$

With the help of (13) the last term may be expressed as

$$
\delta \oint_{0}^{\mathcal{L}_{p}} \omega_{1 m} d s_{m}=\delta \oint_{0}^{\mathcal{L}_{p}} \tau_{m} d s_{m}
$$

Summing up over all the rods, we compute the elastic energy of the bundle

$$
E=\frac{1}{p} \iint_{\Psi} E_{m} d \sigma=E_{\text {bend }}+E_{\text {tors }} .
$$

The integral is divided by the winding number $p$, because, when integrating over the cross-sectional area, each component is counted $p$ times. 
Since the total torsion is conserved for every component rod, the variation of $E$ may be expressed as

$$
\delta E=\pi b \delta \oint_{0}^{\mathcal{L}} f\left(\varkappa^{2}\right) d s+c \iint_{\Psi} \omega_{1 m} d \sigma \delta \oint_{0}^{\mathcal{L}} \tau d s
$$

and $\delta E$ vanishes if there exists $q \neq 0$ such that $\lambda_{1}=q b \pi$ and $\lambda_{2}=q c \iint_{\Psi} \omega_{1 m} d \sigma$, because we assume that the centreline is an extremal for (12). Thus, we have proved the generalization of Theorem 5 from [23] which can be formulated as follows:

For any closed extremal $\mathbf{r}(s)$ of the functional (12) with writhe $\mathcal{W} r(\mathbf{r})=n / 6$, $n \in \mathbb{Z}$, and with the curvature $\varkappa(s) \leq R^{-1}$ for some positive $R$, there exists a parallel bundle in equilibrium with axis $\mathbf{r}$ and with circular cross-section of radius $R$ made up with thin elastic rods. The winding number $p$ is determined by the fractional part of the writhe. The distribution of twist rates of the rods satisfies the conditions

$$
\frac{c \iint_{\Psi} \omega_{1 m} d \sigma}{\pi b}=\frac{\lambda_{2}}{\lambda_{1}} \quad \text { and } \quad p \omega_{1 m} \mathcal{L}=p \oint_{0}^{\mathcal{L}} \tau d s+2 \pi j_{m}, \quad j_{m} \in \mathbb{Z} .
$$

The results of this section may be extended to open bundles provided the boundary conditions are properly specified at the ends. Consider a centreline of a bundle of some fixed length. We want to study an equilibrium of a part of the bundle constrained by two planes orthogonal to the centreline at its ends. Clearly, variation of the shape of the centreline generically implies variation of the lengths of the pieces of other components constrained between the end planes. Still, the volume of the constrained part remains constant. This means that the total length of all the strands in this part does not change. Thus, if we impose the boundary conditions that allow for some strands to shorten and for others to extend under constraint of the fixed sum of their total lengths, then all the above reasoning will be valid for such open bundles.

As a mechanical macroscopic model, we may suggest to put pieces of the bundle at some length near its ends into sleeves of fixed shape that prevent variation of the centrelines of all the rods, yet allow the relative sliding of the strands except the core strand. Anchoring end conditions would be specified for the latter. If the core strand does not exist (like in figures 6 or 7 in contrast to the bundle in figure 5), then the physical implementation of the total length constraint becomes more complicated. One way to overcome this difficulty is to close the component that surrounds the axis of the bundle. Then the strands of this component would have a freedom to slide with respect to each other but its length would remain fixed.

\section{Equilibrium equations}

In this section we first examine the equations that describe the extremals of (12) and then we present the equations for the axis coordinates in space.

\subsection{Equations for the curvature and torsion of the axis}

The Euler-Lagrange equations for (12) may be obtained by adapting equations (77), (78) [8], which are valid for more general functional $\int \phi(\varkappa, \tau) d s$, to our case with 


$$
\begin{aligned}
\phi(\varkappa, \tau)=\lambda_{1} f\left(\varkappa^{2}\right) & +\lambda_{2} \tau+\lambda_{3}: \\
& \lambda_{1}\left\{8 \varkappa^{3} \varkappa_{s}^{2} f^{\prime \prime \prime}+4 \varkappa\left(\varkappa \varkappa_{s s}+3 \varkappa_{s}^{2}\right) f^{\prime \prime}+2\left[\varkappa_{s s}+\varkappa\left(\varkappa^{2}-\tau^{2}\right)\right] f^{\prime}-\varkappa f\right\}+ \\
& +\lambda_{2} \varkappa \tau-\lambda_{3} \varkappa=0, \\
& \lambda_{1}\left[8 \varkappa^{2} \varkappa_{s} \tau f^{\prime \prime}+2\left(2 \varkappa_{s} \tau+\varkappa \tau_{s}\right) f^{\prime}\right]-\lambda_{2} \varkappa_{s}=0,
\end{aligned}
$$

where the prime ${ }^{\prime}$ denotes the derivative with respect to the squared curvature $u \equiv \varkappa^{2}$ : $f^{\prime}=\frac{d f(u)}{d u}$. We can reduce the order of the above system by using two first integrals of (14), (15) that correspond to the Casimirs of the Euclidean group.

Let $\mathbf{F}(v)$ and $\mathbf{M}(v)$ be the internal force and the moment with which the part of the bundle with the axis coordinates $s \leq v$ acts on the part $s>v$. The internal force of the bundle is a constant vector

$$
\mathbf{F}=\left[\lambda_{1}\left(2 \varkappa^{2} f^{\prime}-f\right)-\lambda_{3}\right] \mathbf{T}+2 \lambda_{1} \varkappa_{s}\left(f^{\prime}+2 \varkappa^{2} f^{\prime \prime}\right) \mathbf{N}+\varkappa\left(2 \lambda_{1} \tau f^{\prime}-\lambda_{2}\right) \mathbf{B}=\mathbf{c o n s t}
$$

and its square is the first Casimir

$$
\left[\lambda_{1}\left(2 \varkappa^{2} f^{\prime}-f\right)-\lambda_{3}\right]^{2}+4 \lambda_{1}^{2} \varkappa_{s}^{2}\left(f^{\prime}+2 \varkappa^{2} f^{\prime \prime}\right)^{2}+\varkappa^{2}\left(2 \lambda_{1} \tau f^{\prime}-\lambda_{2}\right)^{2}=F^{2}=\text { const. }
$$

Projection of the moment $\mathbf{M}=-\left(\lambda_{2} \mathbf{T}+2 \lambda_{1} \varkappa f^{\prime} \mathbf{B}\right)$ onto the direction of the internal force provides the second Casimir

$$
\frac{\mathbf{M} \cdot \mathbf{F}}{F}=M_{z}=\text { const }
$$

which may be given the explicit form

$$
4 \lambda_{1} \varkappa^{2} \tau\left(f^{\prime}\right)^{2}-\lambda_{2} f=c=\text { const. }
$$

Expressing the torsion from (17), substituting it into (16) and setting $u=\varkappa^{2}$ results in the equation

$\left(2 \lambda_{1} f^{\prime}\right)^{2}\left(f^{\prime}+2 u f^{\prime \prime}\right)^{2}\left(\frac{d u}{d s}\right)^{2}=4 u\left(f^{\prime}\right)^{2}\left[F^{2}-\left(\lambda_{1}\left(2 u f^{\prime}-f\right)-\lambda_{3}\right)^{2}\right]-\left[c-\lambda_{2}\left(2 u f^{\prime}-f\right)\right]^{2}$,

which can be integrated by quadrature.

Note that if we set $f(u)=u$, which corresponds to the thin elastic rod model, then we come to the equation

$$
4 \lambda_{1}^{2}\left(\frac{d u}{d s}\right)^{2}=4 F^{2} u-4\left(\lambda_{1} u-\lambda_{3}\right)^{2} u-\left(c-\lambda_{2} u\right)^{2}
$$

(cf. equation (16) in Ref. [30]‡).

It is mentioned in Ref. [8] that, for a general functional $\int \phi(\varkappa, \tau) d s$, integrability does not persist. The above consideration shows that if the torsion enters the functional as a separate linear term, then the equations can still be reduced to a quadrature. The case of the perfect bundle with circular cross-section delivers a particular example of such a functional.

$\ddagger$ The preceding equation (15) in Ref. [30] is written for the curvature itself and it should be the same as equation (87) in Ref. [8], but the latter is evidently mistyped: the term in the second parentheses must be $2(\alpha \mu-F J)-\alpha \kappa^{2}$. 


\subsection{Equations for the coordinates of the axis}

Solution of (18) and then (17) gives us the functions of curvature and torsion but does not provide the knowledge of the coordinates of the axis $\mathbf{r}$ in space. A straightforward method to find them is to integrate the Serret-Frenet equations together with the equation

$$
\frac{d \mathbf{r}}{d s}=\mathbf{T} .
$$

In case of the thin rod, it is known that there exist special cylindrical coordinates $\{\rho, \theta, z\}$ in which the centreline is expressed in an especially convenient way $[29,21,22]$. As shown in Ref. [8], this remains valid in the general case.

We direct the $z$-axis along the force vector $\mathbf{F} \neq \mathbf{0}$ and the polar angle $\theta$ is measured from the axis $\mathbf{M} \times \mathbf{F}$. The radial distance is given by

$$
\rho^{2}=\frac{4 \lambda_{1}^{2} \varkappa^{2}\left(f^{\prime}\right)^{2}+\lambda_{2}^{2}-M_{z}^{2}}{F^{2}}
$$

where $M_{z}$ is the constant $z$-component of the moment $\mathbf{M}$. To find the other coordinates one needs to integrate

$$
\frac{d z}{d s}=\frac{1}{F}\left[\lambda_{1}\left(2 \varkappa^{2} f^{\prime}-f\right)-\lambda_{3}\right]
$$

and

$$
\frac{d \theta}{d s}=-\frac{1}{F \rho^{2}}\left(M_{z} \frac{d z}{d s}+\lambda_{2}\right) .
$$

The equations (19), (20), (21) generalize Ilyukhin's equations for the thin elastic rod to the parallel bundles $[21,22]$.

\section{Concluding remarks}

As shown in this work, features of universal geometric nature play essential part in formation of the architecture of densely packed filaments, influencing their conformational energy. The following several comments emphasize the interrelations between the parallel bundles and other models and observations.

Under certain conditions, the DNA toroids may deform taking on a warped shape [12]. Generally, this deformation affects the interstrand distances and the interaction energy between strands. This effect may influence the twist-bend instability of the DNA condensates [28]. However, figure 6 shows a perfectly packed structure with an overall shape that closely resembles the warped toroids in [12]. Thus, keeping interstrand distances constant is compatible with the deformation of the toroid as a whole. Moreover, exluding trivial cases, a parallel bundle should writhe to close to itself.

It is well established that DNA inside a viral capsid is packed at liquid crystal density [9]. We have seen that two requirements: of the continuous hexagonal packing of tubes and of the closedness of the bundle (or its self-touching) lead to three-dimensional structures which are highly interlinked in most cases. Analysis of entanglement is beyond the scope of the present article. It can be only mentioned here that the DNA densely packed in phage capsids appears to be highly knotted [3, 2], as its can be predicted for its near-optimal packing geometry. 
To describe the elastic energy of packed DNA, the thin elastic rod model is applied in Ref. [27] where this energy was represented as a linear combination of two terms: first containing the squared curvature and second the squared (geometrical) torsion. However, it is well known that the thin rod theory (which has become conventional for free DNA) does not include the squared torsion term; instead, there is the squared tangential projection of the Darboux vector, and the equilibria of the rod correspond to extremals of a functional that involves not squared but just linear torsion [30, 23].

On the other hand, the Frank-Oseen energy contains the twist term which generally involves the integral of the squared torsion and, if the cholesteric interaction is taken into account, the total torsion, as well. Note that the perfect hexagonal packing is twistless and implies absence of any twist term in the Frank-Oseen energy caused by interactions of neighbouring polymers, though the individual molecules may still be twisted and their torsional elastic energy may be well caught by the thin rod model. This remark is not limited to perfect hexagonal packing. The case of high density condensates of semiflexible polymers (e.g. DNA) which are long and stiff, is a meeting point of two different descriptions and one always should clearly distinguish which term comes from which model.

DNA is a chiral molecule, therefore one expects that it forms liquid crystals in which the neighbouring polymers are arranged at a cholesteric angle of particular sign. Clearly, no perfect hexagonal packing is possible in this case. It was however found that DNA could form achiral line hexatic phases [46]. This observation is in accordance with the above analysis of the perfect hexagonal order of the twistless bundles. It should be stressed here that these bundles (and hence, the rods in them) may have handedness defined by the sign of its writhe. In other words, chirality may be present in the structures where all the molecules are arranged parallelly to their neighbours.

A macroscopic model of a perfectly packed bundle may be implemented as a set of elastic rods put into a sleeve tight enough to prevent possible cross-overs. Another possibility is to replace the sleeve with a number of discs equally distributed along the bundle and having hexagonally arranged holes for the rods to keep them at equal distances with respect to each other. The discs would then serve as a material realization of the orthogonal cross-sections.

\section{Acknowledgments}

Most of this work was carried out during the author's stay at the Max Planck Institute for Physics of Complex Systems, Dresden, Germany. The author is pleased to thank the Max Planck Society for support.

\section{References}

[1] J. Arsuaga, R. K.-Z. Tan, M. Vazquez, D. W. Sumners, and S. C. Harvey. Investigation of viral DNA packaging using molecular mechanics models. Biophysical Chemistry, 101-102:475-484, 2002.

[2] J. Arsuaga, M. Vazquez, P. McGuirk, S. Trigueros, D. W. Sumners, and J. Roca. DNA knots reveal a chiral organization of DNA in phage capsids. Proc. Natl. Acad. Sci. USA, 102(26):9165-9169, 2005.

[3] J. Arsuaga, M. Vázquez, S. Trigueros, D. W. Sumners, and J. Roca. Knotting probability of DNA molecules confined in restricted volumes: DNA knotting in phage capsids. Proc. Natl. Acad. Sci. USA, 99(8):5373-5377, 2002. 
[4] A. Bezdek and W. Kuperberg. Maximum density space packing with congruent circular cylinders of infinite length. Mathematika, 37:74-80, 1990.

[5] R. L. Bishop. There is more than one way to frame a curve. Amer. Math. Monthly, 82(3):246251, 1975.

[6] C. Böttcher, C. Endisch, J.-H. Fuhrhop, C. Catterall, and M. Eaton. High-yield preparation of oligomeric C-type DNA toroids and their characterization by cryoelectron microscopy. Journal of the American Chemical Society, 120(1):12-17, 1998.

[7] D. Bray. Cell Movements: From Molecules to Motility. Garland Publishing, 2 edition, 2001.

[8] R. Capovilla, C. Chryssomalakos, and J. Guven. Hamiltonians for curves. J. Phys. A: Math. Gen., 35(31):6571-6587, 2002.

[9] M. E. Cerritelli, N. Cheng, A. H. Rosenberg, C. E. McPherson, F. P. Booy, and A. C. Steven. Encapsidated conformation of bacteriophage T7 DNA. Cell, 91:271-280, 1997.

[10] D. Durand, J. Doucet, and F. Livolant. A study of the structure of highly concentrated phases of DNA by X-ray diffraction. Journal de Physique II, 2(9):1769-1783, 1992.

[11] W. C. Earnshaw and S. C. Harrison. DNA arrangement in isometric phage heads. Nature, 268:598-602, 1977.

[12] W. C. Earnshaw, J. King, S. C. Harrison, and F. A. Eiserling. The structural organization of DNA packaged within the heads of T4 wild-type, isometric and giant bacteriophages. Cell, 14:559-568, 1978.

[13] Yu. M. Evdokimov, A. L. Platonov, A. S. Tikhonenko, and Ya. M. Varshavsky. A compact form of double-stranded DNA in solution. FEBS Letters, 23(2):180-184, 1972.

[14] M. D. Frank-Kamenetskii and A. V. Vologodskii. Topological aspects of the physics of polymers: The theory and its biophysical applications. Soviet Physics - Uspekhi, 24(8):679-696, 1981.

[15] F. B. Fuller. The writhing number of a space curve. Proc. Natl. Acad. Sci. USA, 68(4):815-819, 1971.

[16] F. B. Fuller. Decomposition of the linking number of a closed ribbon: A problem from molecular biology. Proc. Natl. Acad. Sci. USA, 75(8):3557-3561, 1978.

[17] R. Golan, L. I. Pietrasanta, W. Hsieh, and H. G. Hansma. DNA toroids: stages in condensation. Biochemistry, 38(42):14069-14076, 1999.

[18] S. C. Harrison. Packaging of DNA into bacteriophage heads: A model. J. Mol. Biol., 171:577$580,1983$.

[19] H. Hess, J. Clemmens, C. Brunner, R. Doot, S. Luna, K.-H. Ernst, , and V. Vogel. Molecular self-assembly of "nanowires" and "nanospools" using active transport. Nano Lett., 5(4):629633, 2005.

[20] N. V. Hud and K. H. Downing. Cryoelectron microscopy of lambda phage DNA condensates in vitreous ice: The fine structure of DNA toroids. Proc. Natl. Acad. Sci. USA, 98(26):1492514930, 2001.

[21] A. A. Ilyukhin. On the deformation of the elastic curve [o deformacii uprugoj linii]. Mekhanika tverdogo tela, 1:128-138, 1969. [in Russian].

[22] A. A. Ilyukhin. Spatial Problems of the Nonlinear Theory of Elastic Rods [Prostranstvennye Zadachi Nelinejnoj Teorii Uprugih Sterzhnej]. Naukova Dumka, Kiev, 1979. [in Russian].

[23] T. A. Ivey and D. A. Singer. Knot types, homotopies and stability of closed elastic rods. Proceedings of the London Mathematical Society, 79(3):429-450, 1999.

[24] R. D. Kamien. The geometry of soft materials: a primer. Reviews of Modern Physics, 74(4):953971, 2002.

[25] J. Kindt, S. Tzlil, A. Ben-Shaul, and W. M. Gelbart. DNA packaging and ejection forces in bacteriophage. PNAS, 98(24):13671-13674, 2001.

[26] M. Kleman and O. D. Lavrentovich. Soft Matter Physics. Springer, 2003.

[27] W. S. Klug and M. Ortiz. A director-field model of DNA packaging in viral capsids. Journal of the Mechanics and Physics of Solids, 51(10):1815-1847, 2003.

[28] I. M. Kulić, D. Andrienko, and M. Deserno. Twist-bend instability for toroidal DNA condensates. Europhysics Letters, 67(3):418-424, 2004.

[29] L. D. Landau and E. M. Lifshitz. Theory of Elasticity, volume 7 of Course of Theoretical Physics. Pergamon, Oxford, 2 edition, 1970.

[30] J. Langer and D. A. Singer. Lagrangian aspects of the Kirchhoff elastic rod. SIAM Review, 38(4):605-618, 1996.

[31] J. Lepault, J. Dubochet, W. Baschong, and E. Kellenberger. Organization of double-stranded DNA in bacteriophages: a study by cryo-electron microscopy of vitrified samples. The EMBO Journal, 6(5):1507-1512, 1987.

[32] F. Livolant and A. Leforestier. Condensed phases of DNA: Structures and phase transitions. Progress in Polymer Science, 21(6):1115-1164, 1996. 
[33] F. Livolant, A. M. Levelut, J. Doucet, and J. P. Benoit. The highly concentrated liquidcrystalline phase of DNA is columnar hexagonal. Nature, 339:724-726, 1989.

[34] A. C. Maggs. Writhing geometry at finite temperature: Random walks and geometric phases for stiff polymers. J. Chem. Phys., 114(13):5888-5896, 2001.

[35] D. Marenduzzo and C. Micheletti. Thermodynamics of DNA packaging inside a viral capsid: The role of DNA intrinsic thickness. Journal of Molecular Biology, 330(3):485-492, 2003.

[36] K. A. Marx and G. C. Ruben. Evidence for hydrated spermidine-calf thymus DNA toruses organized by circumferential DNA wrapping. Nucleic Acids Res., 11(6):1839-1854, 1983.

[37] R. Metzler and P. G. Dommersnes. Helical packaging of semiflexible polymers in bacteriophages. European Biophysics Journal, 33(6):497-505, 2004.

[38] S. Y. Park, D. Harries, and W. M. Gelbart. Topological defects and the optimum size of DNA condensates. Biophysical Journal, 75(2):714-720, 1998.

[39] P. K. Purohit, J. Kondev, and R. Phillips. Mechanics of DNA packaging in viruses. Proc. Natl. Acad. Sci. USA, 100(6):3173-3178, 2003.

[40] S.C. Riemer and V.A. Bloomfield. Packaging of DNA in bacteriophage heads: Some considerations on energetics. Biopolymers, 17(3):785-794, 1978.

[41] N. C. Seeman and P. S. Lukeman. Nucleic acid nanostructures: bottom-up control of geometry on the nanoscale. Reports on Progress in Physics, 68(1):237-270, 2005.

[42] J. H. Shin, L. Mahadevan, P. T. So, and P. Matsudaira. Bending stiffness of a crystalline actin bundle. Journal of Molecular Biology, 337(2):255-261, 2004.

[43] E. L. Starostin. On the writhe of non-closed curves, 2002. arXiv: physics/0212095, 56 p.

[44] E. L. Starostin. In: Physical and Numerical Models in Knot Theory Including Applications to the Life Sciences, eds. J. A. Calvo, K. C. Millett, E. J. Rawdon and A. Stasiak, volume 36 of Series on Knots and Everything, chapter On the writhing number of a non-closed curve, pages 525-545. World Scientific, 2005.

[45] E. L. Starostin. On the number of tubes touching a sphere or a tube. Geometriae Dedicata, 2005. In press. Preprint mpi-pks/0408004 (2004).

[46] H. H. Strey, J. Wang, R. Podgornik, A. Rupprecht, L. Yu, V. A. Parsegian, and E. B. Sirota. Refusing to twist: Demonstration of a line hexatic phase in DNA liquid crystals. Phys. Rev. Lett., 84(14):3105-3108, 2000.

[47] A. Thess, R. Lee, P. Nikolaev, H. Dai, P. Petit, J. Robert, C. Xu, Y. H. Lee, S. G. Kim, A. G. Rinzler, D. T. Colbert, G. E. Scuseria, D. Tomanek, J. E. Fischer, and R. E. Smalley. Crystalline ropes of metallic carbon nanotubes. Science, 273(5274):483-487, 1996.

[48] L. G. Tilney. Actin filaments in the acrosomal reaction of Limulus sperm. Motion generated by alterations in the packing of the filaments. J. Cell Biol., 64(2):289-310, 1975.

[49] A. A. Travers and J. M. T. Thompson. An introduction to the mechanics of DNA. Philosophical Transactions: Mathematical, Physical and Engineering Sciences, 362(1820):1265-1279, 2004.

[50] J. Ubbink and T. Odijk. Polymer- and salt-induced toroids of hexagonal DNA. Biophysical J., 68:54-61, 1995.

[51] J. Widom and R. L. Baldwin. Tests of spool models for DNA packaging in phage lambda. J. Mol. Biol., 171(4):419-437, 1983. 